\title{
Alterations in mitochondrial function and energy metabolism- related properties in thyroid cancer stem cells
}

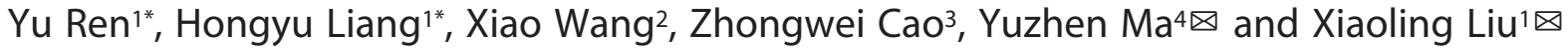 \\ 1Scientific Research Department Center, Inner Mongolia People's Hospital, Hohhot, Inner Mongolia Autonomous Region, 010017 , China; ${ }^{2} \mathrm{Clinical}$ \\ Medical Research Center, Inner Mongolia People's Hospital, Hohhot, Inner Mongolia Autonomous Region, 010017, China; ${ }^{3 T h y r o i d, ~ M a m m a r y, ~}$ \\ and Hernia surgery Department, Inner Mongolia People's Hospital, Hohhot, Inner Mongolia Autonomous Region, 010017, China; ${ }^{2}$ Reproductive \\ Medicine Centre, Inner Mongolia People's Hospital, Hohhot, Inner Mongolia Autonomous Region, 010017, China
}

Increasing evidence indicates that cancer stem cells (CSCs) are initiators of the occurrence, development, and recurrence of malignant tumors. Mitochondria are important organelles in eukaryotic cells, not only responsible for converting part of energy released during nutrients oxidation into the energy-yielding molecule adenosine triphosphate (ATP) to fuel the activities of cell, but also play essential roles in processes such as cell apoptosis and cellular proliferation. The mitochondrialrelated abnormalities have also been considered to have an important role in the origin and development of tumors. This study aimed at testing the abnormalities in mitochondrial function and energy/metabolism-related phenotypes in thyroid cancer stem cells (TCSCs). TCSCs were isolated and identified from MDA-T32 thyroid carcinoma cell line. The mitochondrial mass and mitochondrial arrangement, amount of mitochondrial DNA (mtDNA), mitochondrial membrane potential (MMP), oxygen/glucose consumption, and intracellular concentrations of reactive oxygen species (ROS) and ATP levels were examined. Perinuclear mitochondrial distribution, low amount of mtDNA and oxygen/glucose consumption, high MMP, and low intracellular ROS and ATP concentrations were observed in TCSCs. Alterations in mitochondrial function and cellular energy metabolism may be used as novel indicators of thyroid cancer.

Key words: TCSCs, papillary thyroid carcinoma, MDA-T32 cells, cancer stem cell, side population, mitochondrial membrane potential

Received: 18 May, 2020; revised: 12 July, 2021; accepted: 21 October, 2021; available on-line: 26 November, 2021

凶e-mail: YuzhenMadhj@163.com (Y. M); XiaolingLiudhj@163.com (X. L)

Acknowledgements of Financial Support: This work was sponsored by the Inner Mongolia Autonomous Region Science and Technology Project (No. 201702118).

*These authors contribute equally to this study

Abbreviations: CSCs, cancer stem cells; ATP, adenosine triphosphate; TCSCs, thyroid cancer stem cells; mtDNA, mitochondrial DNA; MMP, mitochondrial membrane potential; ROS, reactive oxygen species; TC, thyroid cancer; DMEM, Dulbecco's Modified Eagle medium; FBS, fetal bovine serum; PBS, phosphate buffer solution; SP, side population; PTPC, permeability transition pore complex; UCP, uncoupling proteins.

\section{INTRODUCTION}

Thyroid cancer (TC) is the most frequently diagnosed endocrine cancer and causes deaths (Lin, 2011); it is the fifth most common malignancy among women in the United States (Hernandez et al., 2010). Approximately $10-20 \%$ of patients with papillary thyroid car- cinoma stages I or II have disease recurrence (Toniato et al., 2008). Many studies have indicated the important role of cancer stem cells (CSCs) in cancer occurrence, development, and recurrence (Visvader et al., 2008). Thyroid cancer stem cells (TCSCs) are reported to have the ability to sustain neoplastic growth owing to their resistance to chemotherapy and radiation therapy, which is a major obstacle for the effective treatment of TC (Lin et al., 2013).

Mitochondria, the primary energy-generating system in most eukaryotic cells, are the most important organelles that provide energy to sustain the metabolic needs of eukaryotic cells involved in intermediary metabolism, calcium signaling, proliferation, and apoptosis (Chan, 2006). In addition, mitochondria can regulate cellular metabolism by affecting the biosynthesis of steroid hormones and porphyrin, lipid and urea cycle metabolism, and amino acid interconversion (Brookes et al., 2004). Perinuclear mitochondrial arrangement, reduced number of mitochondria, low amount of mitochondrial DNA (mtDNA), and low adenosine triphosphate (ATP) levels are considered indicators of "stemness" of undifferentiated cells (Xiao-Qun et al., 2011).

Several studies have focused on the relationship between energy metabolism and self-renewal and differentiation potential in stem cells. For example, a previous study indicated that perinuclear mitochondrial distribution, higher mitochondrial membrane potential (MMP), lower amounts of $\mathrm{mtDNA}$, lower oxygen/glucose consumption, and lower amounts of reactive oxygen species (ROS) and ATP were observed in lung CSCs. High levels of ROS are generated by electron transport in the mitochondrial membrane and produced in the thyroid under physiological conditions (Lee et al., 2015). mtDNA mutations are associated with age-dependent tumor progression in the thyroid (Witte et al., 2007). Eosinophilic Hurthle thyroid cancers may have abundant mitochondria, resulting in deep eosinophilic staining characteristics (Straccia et al., 2019). Cancer cells have higher glucose consumption and lactate production than normal cells, even under normoxic conditions; this is known as the Warburg effect (Chiaradonna et al., 2006, Ward et al., 2012). In addition, a severe mitochondrial OxPhos defects are present in many cancer cells, probably caused by mutations in mtDNA and abnormal expression of relevant genes (Chiaradonna et al., 2012). These results suggest a strong correlation between CSCs, mitochondrial function, and energy/metabolism.

Based on these investigations, we hypothesized that abnormal mitochondrial function and alterations in energy/metabolism-related phenotypes occur in TCSCs, 
which could contribute to a more comprehensive understanding of the pathogenesis and progression of thyroid cancer. In this study, TCSCs were isolated and identified in the MDA-T32 cell line, and some of the indicators of "stemness" of undifferentiated cells were examined.

\section{MATERIALS AND METHODS}

\section{Cell line and sphere culture}

The human papillary thyroid carcinoma cell line MDA-T32 was seeded in 6-well plates (1000 cells/well) with Dulbecco's Modified Eagle medium (DMEM; Hyclone, Utah, USA) supplemented with 10\% fetal bovine serum (FBS; Gibco, New York, USA) and cultured at $37^{\circ} \mathrm{C}$ in humidified $5 \% \mathrm{CO}_{2}$ atmosphere with saturated humidity for two weeks; Then, they were observed and their micrographs were captured under an Olympus IMT-2 inverted microscope (San Diego, CA, USA) and a Moticam Prodigital camera (Motic, Seneco, MI, Italy). The selected cells were seeded in 6-well plates $\left(2 \times 10^{4}\right.$ cells/well) and cultured in stem cell culture medium. After approximately 1 week, 20-40 small round clustered cell spheres were observed in each well, and each sphere contained 5-10 cells. The monolayers were observed at the bottom. After 2 weeks, the spheres expanded 10-20 times, and 100-200 cells/ sphere were the first generation of cultured stem cell balls. The spheres were dissociated, and single cells were seeded into a $75-\mathrm{mL}$ flask containing serum-free stem cell medium and cultured for 1-2 weeks for further analysis.

\section{Real-time reverse transcription polymerase chain reaction and polymerase chain reaction (RT-PCR)}

Total RNA was isolated and subjected to reverse transcription using Superscript II (Life Technologies). DNA (including genomic and mitochondrial) was isolated, and RT-PCR was conducted using the SYBR ${ }^{\circledR}$ PrimeScript ${ }^{\text {TM }}$ PCR kit II (TaKaRa, Japan). $\beta$-Actin served as an internal control. Specific primers were obtained from Sangon Biotech (Shanghai, China). The primer sequences were used as follows. CD34-F: GCC ATT CAG CAA GAC AAC AC, CD34-R: CGT TTA CCC AAA GAA GAC CAG; C-KIT-F: TTC CTT GTT GAC CGC TCC TT, c-kit-R: CAG CAC TGA CTT GCC CTC C; Twist1-F: GAC AGT GAT TCC CAG ACG G, Twist1-R: GTC CAT AGT GAT GAA TTT CCT; Sox2-F: ATC ACC CAC AGC AAA TGA CA, Sox2-R: CAA AGC TCC TAC CGT ACC ACT A; Oct4-F: TAT TCA GCC AAA CGA CCA TCT, Oct-R: TCA GCT TCC TCC ACC CAC TT; Nanog-F: AGG CAA ACA ACC CAC TTC T; Nanog-R: TCA CAC CAT TGC TAT TCT TCG; CD133-F: GCA CTC TAT ACC AAA GCG TCA A, CD133-R:CTC CCA TAC TTC TTA GTT TCC TCA; C-myc-F: GGT GGC TAT TCT GCC CAT TT, C-mycR: CGA GGT CAT AGT TCC TGT TGG T; Bmi-1-F: CTC CAC CTC TTC TTG TT'T GC; Bmi-1-R: GAT GAC CCA TTT ACT GAT GAT TT; CD44s-F: CTC CAC CTC T'TC TTG TTT GC, CD44s-R: TCC ACT TGG CTT TCT GTC CTC; MELK-F: TCC TGT TGA GTG GCA AAG CA, MELK-R: GCA GAA GAT AGG TAG CCG TGA G; N-Cadherin-F: ACG CCA GGC CAA ACA ACT T; N-Cadherin-R: TCG TCG GAT TCC CAC AGG; Cox I-F: TGG CCT GAC TGG CAT TGT ATT, Cox I-R: CGG ATA GGC CGA GAA AGT GTT; $\beta$-actin-F: CCT GTA CGC CAA CAC AGT GC, $\beta$-actin-R: ATA CTC CTG CTT GCT GAT CC.

\section{Detection of side population cells}

MDA-T32 cells $\left(1 \times 10^{5}\right)$ were seeded onto coverslips and cultured for three days. After staining with $5 \mu \mathrm{g} /$ mL Hoechst 33342 (Beyotime Co., Nantong, China), the cells were washed twice with phosphate buffer solution (PBS) and analyzed using laser confocal microscopy.

\section{Determination of the distribution and amount of mitochondria}

The cells $\left(5 \times 10^{4}\right)$ were stained with $50 \mathrm{nM}$ MitoTracker Green (Invitrogen, USA) for $15 \mathrm{~min}$ at $37^{\circ} \mathrm{C}$, washed twice with PBS, and analyzed using laser confocal microscopy and flow cytometry (Beckman, USA).

\section{MMP}

The accumulation of rhodamine-123 (Rh123) was proportional to MMP, which was used to measure MMP. The cells $\left(5 \times 10^{4}\right)$ were stained with Rh123 $(1.0 \mu \mathrm{g} / \mathrm{mL}$, Invitrogen) for $20 \mathrm{~min}$ at $37^{\circ} \mathrm{C}$ and analyzed using flow cytometry.

\section{Oxygen consumption}

Mitochondria were obtained using a mitochondrial isolation kit (Beyotime Co.). The cells $\left(1 \times 10^{7}\right)$ were put into $10 \mathrm{~mL}$ glass centrifuge tubes. The collected cell suspension was centrifuged at $1000 \times g$ for 10 minutes, and the supernatant was discarded. The cells were resuspended in $1 \mathrm{ml}$ of PBS pre-cooled in an ice bath and centrifuged at $600 \times g$ for 5 minutes at $4^{\circ} \mathrm{C}$ and the supernatant was removed. $1 \mathrm{ml}$ of mitochondrial separation reagent was added, the cells were gently suspended, and ice bathed for $15 \mathrm{~min}$. The cells were homogenized with a $1 \mathrm{ml}$ glass homogenizer and mitochondria were then separated using the mitochondria isolation kit (Beyotime $\mathrm{Co}$ ). Finally, the mitochondria were dissolved in $150 \mu \mathrm{l}$ detergent. The respiration control rate, the ratio of state $3 /$ state 4 respiratory rate, was calculated.

The Clark-type oxygen electrode used to monitor oxygen consumption for $30 \mathrm{~min}$ at $28^{\circ} \mathrm{C}$ in a preheated cell. The low point of oxygen consumption was adjusted with ferrous sulfate. Added $0.7 \mathrm{ml}$ of mitochondrial reaction medium for $2 \mathrm{~min}$. Added 0.1 $\mathrm{ml}$ of mitochondrial suspension for $1 \mathrm{~min}$ and added 10 ul oxidation substrate (sodium malate and sodium pyruvate are each $0.5 \mathrm{M}$ ) for $1 \mathrm{~min}$. After that, added $5 \mathrm{ml}$ of $0.1 \mathrm{M}$ LADP solution, recorded the oxygen consumption curve and observed the changes in mitochondrial respiratory oxygen consumption rate. From the curve, calculated the oxygen consumption after ADP was added (I respiration, ST3) and after ADP was exhausted (IV respiration, ST4), and expressed by the number of moles of oxygen atoms consumed by unit mitochondria in unit time $(\mathrm{mMO} / \mathrm{min} / \mathrm{mg}$ pro). The ratio of oxygen consumption between state III and state IV (ST3/ST4) represented the respiration control of rate.

\section{Glucose consumption}

The cells were dissociated into single-cell suspensions and seeded in 6-well plates $\left(1 \times 10^{5}\right.$ cells/well $)$. After $24 \mathrm{~h}$ of incubation at $37^{\circ} \mathrm{C}$, the concentration of glucose in the culture medium was measured using a glucose assay kit (Rsbio, Shanghai, China). Glucose consumption was calculated as the glucose concentration before incubation minus the glucose concentration after incubation. 
A
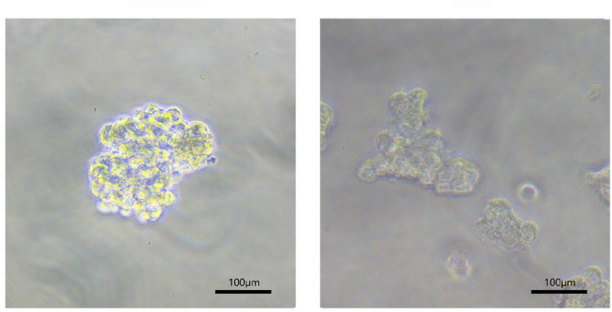

B

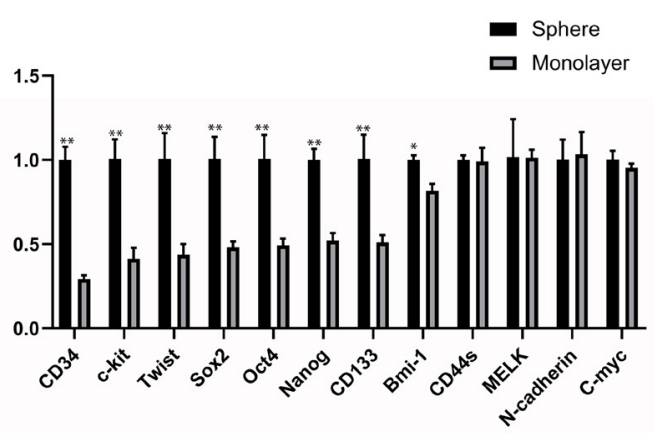

Figure 1. A stem cell-like subpopulation was observed in the MDA-T32 cell line

(A) holoclones (left) and non-holoclones (right) were observed under an inverted microscope. (B) the mRNA expression levels of stem cell-related transcripts, including CD34, c-kit, Twist1, Sox2, Oct4, Nanog, CD133, Bmi-1, CD44s, MELK, N-cadherin, and C-myc, were detected using qRT-PCR.

\section{A}

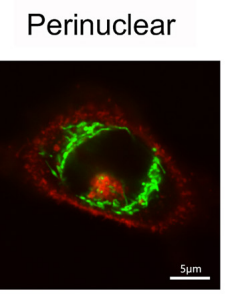

Homogeneous
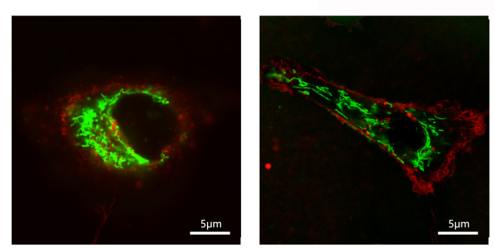

\section{B}

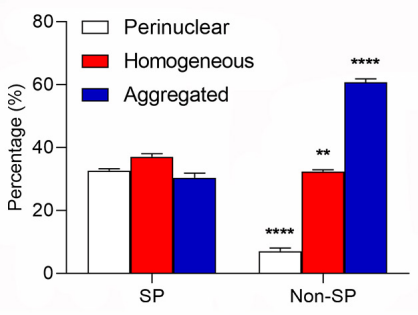

Figure 2. Distribution of mitochondria in SP and non-SP subpopulations of the MDA-T32 cell line

(A) Mitochondria were visualized using MitoTracker Green (green), and the cell periphery was stained using Dil (red). The intensity was detected using laser confocal microscopy, scale bar $=5 \mu \mathrm{m}$. Cells with a predominantly perinuclear arrangement of mitochondria had a high fluorescence intensity near the nucleus, which reduced precipitously toward the cell periphery. (B) Quantification of mitochondrial distribution pattern in SP and non-SP subpopulations of the MDA-T32 cell line. SP, side population. ${ }^{* *} p<0.01$ and ${ }_{* * * *} p<0.0001$ compared to the SP group.

\section{Intracellular ATP concentration}

An ATP assay kit (Beyotime Corporation) was used to detect the intracellular ATP concentration. Briefly, $1 \times 10^{5}$ cells were lysed and centrifuged, and the supernatants were collected. The supernatants were mixed with luciferin-luciferase complex $(100 \mu \mathrm{L})$, and the luminescent signal was measured using a luminometer (Pforzheim, Germany).

\section{Intracellular ROS concentration}

The cells $\left(1 \times 10^{6}\right)$ were stained with10 $\mu \mathrm{M}$ DCF-DA (2',7'-dichlorodihydrofluorescein diacetate, Molecular Probes) to measure intracellular ROS and then analyzed using flow cytometry. The excitation and emission wavelengths were $488 \mathrm{~nm}$ (blue) and $525 \mathrm{~nm}$ (green), respectively.

\section{RESULTS}

\section{A stem cell-like subpopulation was observed in the MDA-T32 cell line}

The human papillary thyroid carcinoma cell line MDA-T32 was seeded in 6-well plates (1000 cells/well) with DMEM, supplemented with 10\% FBS, and cultured at $37^{\circ} \mathrm{C}$ in humidified $5 \% \mathrm{CO}_{2}$ atmosphere with saturated humidity for two weeks, after which the cells, including holoclones and non-holoclones, were observed under an Olympus IMT-2 inverted microscope (Fig. 1A). The mRNA expression levels of stem cell-related transcripts were measured. As shown in Fig. 1B, the mRNA expression levels of CD34, c-kit, Twist1, Sox2, Oct4, Nanog, CD133, and Bmi-1 in spheres were significantly increased compared to those in the monolayer (Fig. 1B).

\section{Perinuclear mitochondrial distribution and mitochondrial mass were higher in MDA-T32 side population cells than in non-SP cells}

TCSCs displayed three distinct spatial arrangements of mitochondria: perinuclear, homogeneous, and aggregated, which were similar to those in the normal adult stem cell lines (Fig. 2A). The mitochondrial distribution pattern in side population $(\mathrm{SP})$ cells was significantly different from that in MDA-T32 non-SP cells (Fig. 2B). Many MDAT32 SP cells showed increased perinuclear mitochondrial distribution, while MDA-T32 non-SP cells displayed aggregated mitochondrial distributions.

As shown by flow cytometry, the mitochondrial mass in spheres was up-regulated with respect to the monolayers. The results demonstrate significant average intensities in the SP and non-SP cells, indicating increased mitochondrial mass in the SP compared to that in nonSP cells (Fig. 3A and 3B). A differentiation medium, DMEM with $10 \% \mathrm{FBS}$, was used to treat the spheres, and the intensity of the Mito-Tracker Green per cell decreased sharply from day 0 to day 1 , increased from day 1 to day 2, and decreased gradually from day 2 to day 3. The mitochondrial mass significantly increased from day 4 to day 8. After 16 days, the mitochondrial mass was down-regulated in the differentiated descendants (Fig. 3C).

\section{Low amount of mtDNA in TCSCs}

The copies of mtDNA, calculated using Cox I/actin, were lower in spheres than in monolayers (Fig. 3D). 
A
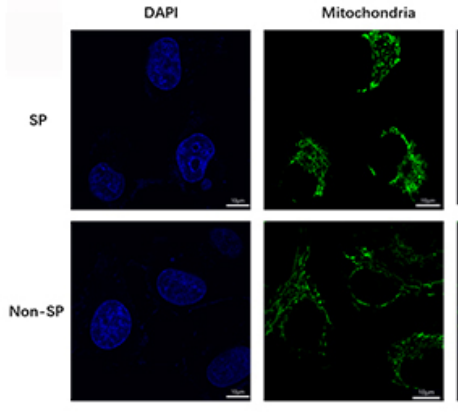

C

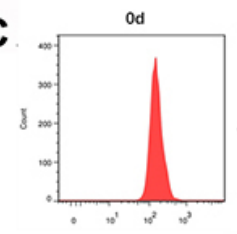

$4 \mathrm{~d}$

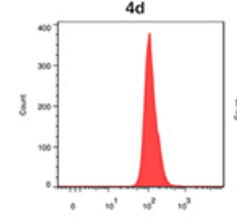

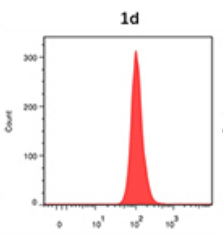

$8 d$

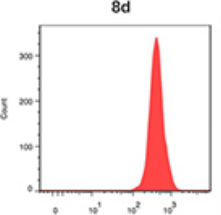

Merge
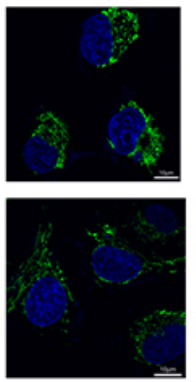

B

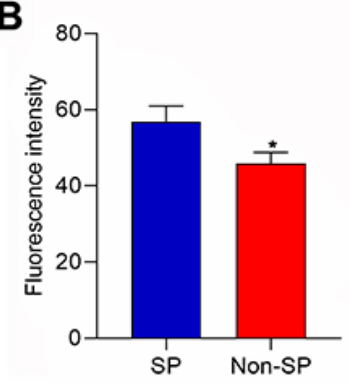

Figure 3. Amount of mitochondria and mtDNA

(A, B) Images and statistical analysis on the average intensities of MitoTracker Green in SP and non-SP subpopulations from the results of laser confocal microscopy. (C) Change in mitochondrial mass during differentiation of the spheres (0-16 days) using flow cytometry. (D) mtDNA copies were presented as Cox/actin, using real-time PCR. ${ }^{* *} p<0.01$ and ${ }^{* * *} p<0.001$ compared to the SP/monolayer group.

A

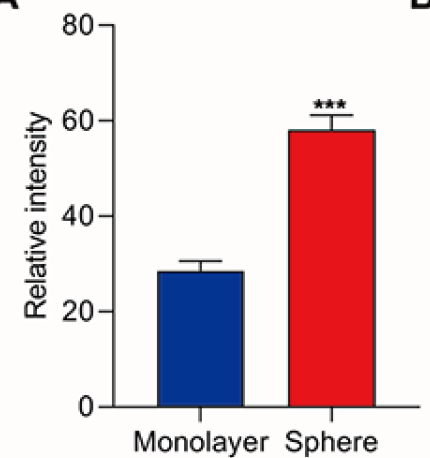

C

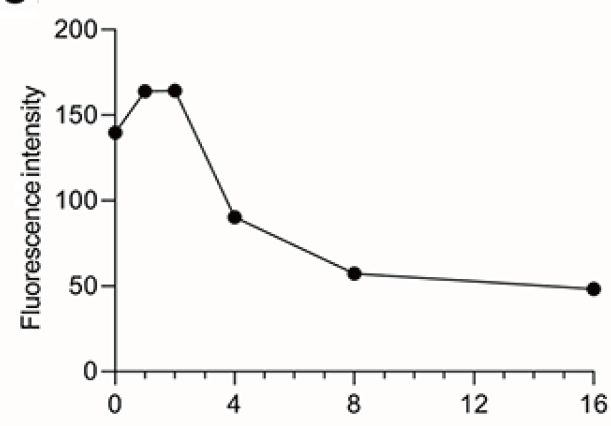

B
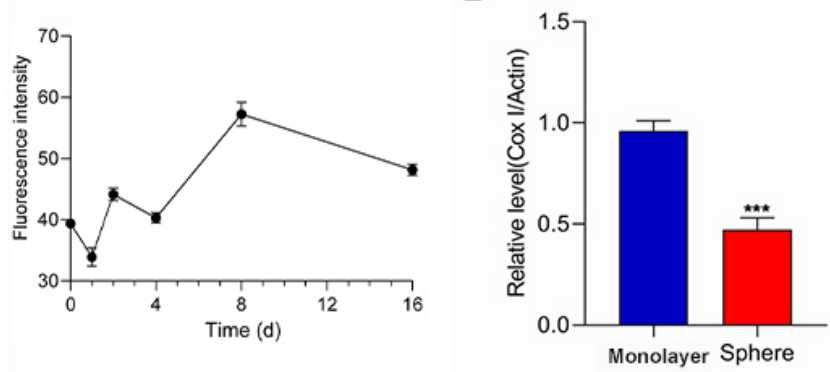

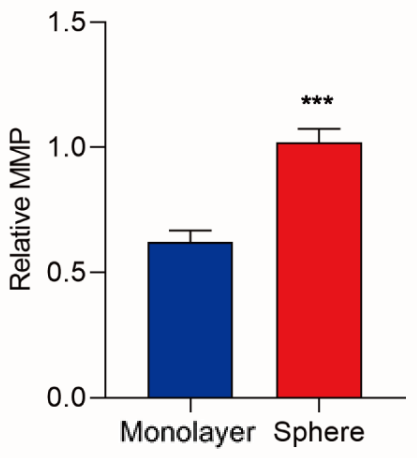

od
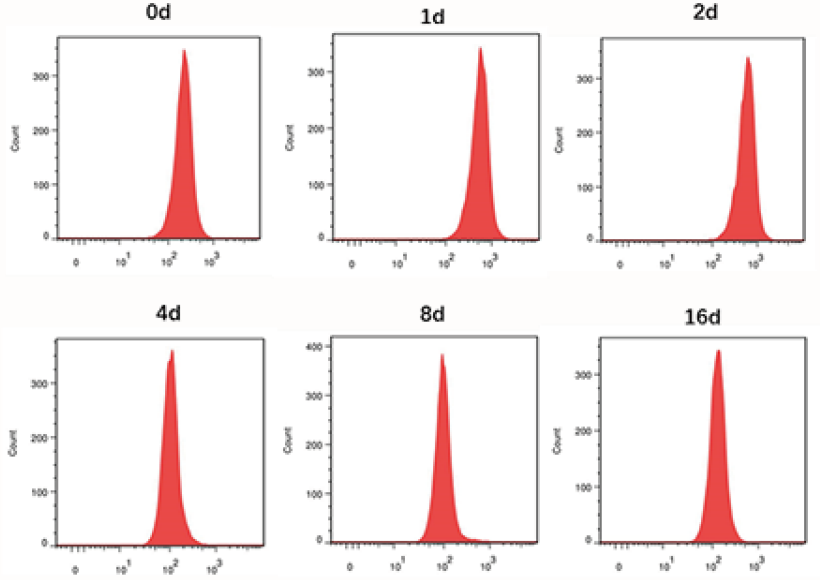

Figure 4. High mitochondrial membrane potential (MMP) was observed in TCSCs

(A) The intensity of Rh123 in spheres and monolayer cells, using flow cytometry. (B) The relative MMP in spheres and monolayer cells.

(C) Change in MMP during differentiation of the spheres. ${ }^{* *} p<0.001$ compared to the monolayer group. 

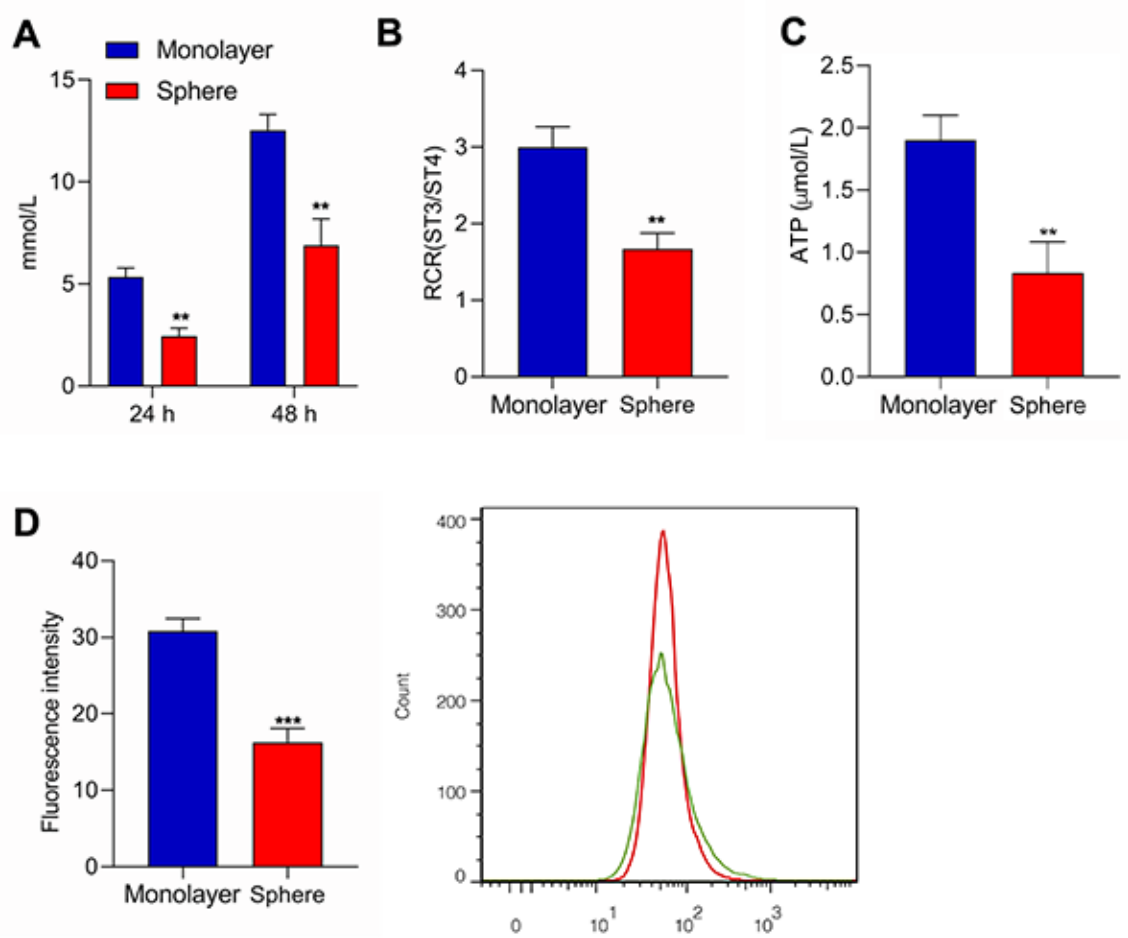

Figure 5. Glucose/oxygen consumption and intracellular ATP/ROS concentration in spheres and monolayer cells

(A) Glucose consumption in spheres and monolayer cells. (B) Oxygen consumption in spheres and monolayer cells. The ratio of oxygen consumption between state III and state IV (ST3/ST4) represented the respiration control of rate (RCR). (C) ATP concentration per cell. (D) Intracellular ROS concentration was detected using flow cytometry. Red was presented as sphere and green was presented as monolayer. ${ }^{* * *} p<0.001$ compared to the monolayer group.

\section{Higher MMP was observed in TCSCs}

The MMP levels in spheres and monolayers were detected. The results showed that the MMP level in spheres was higher than that in monolayers. The intensity of Rh123 was dependent on MMP level and mitochondrial mass. Thus, the ratio of the intensity of Rh123 and mitochondrial mass was calculated to show the relative MMP level. The results suggested that the relative MMP level in spheres was higher than that in monolayers (Fig. 4A and 4B). A differentiation medium, DMEM with $10 \%$ FBS, was used to treat the spheres. On the first day, the intracellular intensity of Rh123 increased significantly. The MMP level plateaued between days 1 and 2 and decreased rapidly until day 16 (Fig. 4C).

\section{TCSCs consume less glucose and oxygen and have lower ATP and ROS concentrations than non-TCSCs}

The spheres consumed less glucose and oxygen than the monolayers (Fig. 5A and 5B). As shown in Fig. 5C, the intracellular ATP concentration, depicted as ATP content per cell, was lower in spheres than in monolayers. In addition, considerably lower levels of ROS were observed in the sphere than in the monolayer (Fig. 5D).

\section{DISCUSSION}

CSCs have specific features, including cytotoxic resistance, immunogenicity, invasiveness, hypoxic tolerance, proliferation/division, and the capacity for neovessel induction (Xiao-Qun et al., 2011). Metabolism is an important characteristic of CSC energy properties and can be used as a diagnostic and therapeutic target.

In our study, TCSCs were isolated and identified in the MDA-T32 cell line using the mRNA expression lev- els of stem cell-related transcripts. The mRNA expression levels of CD34, c-kit, Twist, Sox2, Oct4, Nanog, CD133, and Bmi-1 in spheres were significantly increased compared to those in monolayer. TCSCs might be isolated according to the presence of cell surface markers including CD133 and CD34; putative stem cells markers such as Oct4, Sox2, and Nanog; normal adult stem cell molecules related to stemness such as c-kit and Bmi-1; and EMT transcription factors such as Twist1. Notably, these markers and factors are closely associated with the cellular metabolic pathway of mitochondria and energy. CD34 can sustain low oxygen consumption and ATP levels, two specific mitochondrial features, in human hematopoietic stem cells (Piccoli et al., 2005). A previous study indicated that during the differentiation of embryonic stem cells, significant alterations in oxygen consumption, mtDNA copy number, mitochondrial morphology, and ATP levels are accompanied by the decreased expression of Oct4, Sox2, and Nanog (Facucho-Oliveira et al., 2009). Liu and others (Liu et al., 2009) reported that Bmil can maintain mitochondrial function and redox homeostasis (Liu et al., 2009). Pan and others (Pan et al., 2009) found that twist-1, selectively expressed in adipose tissues, can interact with PGC- $1 \alpha$ to inhibit the metabolism and uncoupling of mitochondria (Pan et al., 2009).

In this study, three types of mitochondrial arrangements and significant differences in mitochondrial arrangement patterns were observed. Lonergan et al. reported that the percentage of cells with a perinuclear mitochondrial arrangement, low rate of oxygen consumption, low ATP/cell content, and low mtDNA content may be indicators of stem cell differentiation competence, which might serve as an indicator of stem cells (Lonergan et al., 2006; Lonergan et al., 2007). Also, many 
benefits were reported about the content between mitochondria and nuclei, such as cellular material transport, energy transfer. Thus, it indicated that a perinuclear mitochondrial arrangement could help the differentiation of CSCs (Piccoli et al., 2005).

In our study, MDA-T32 spheres consistently had significantly lower mtDNA copies than monolayers, and the quantity of mtDNA content increased during the differentiation of MDA-T32 spheres. These results suggest that mtDNA replication is extensively enhanced.

MMP reflects the functional status of the mitochondria and is correlated with cell differentiation, tumor development, and progression. In the present study, we showed that a higher MMP was observed in TCSCs. Bonnet and others (Bonnet et al., 2007) found that several human cancer cells, including glioblastoma M059K, lung cancer A549, and breast cancer MCF-7 cells, have higher MMP than normal cells, thus contributing to apoptosis resistance (Bonnet et al., 2007). It has also been reported that prostate cancer and melanomas present a higher MMP than normal epithelial cells (Kroemer et al., 2008). The possible mechanisms should be considered, as follows: (1) Overexpression of bcl-2. The bcl-2 family member proteins play a role by interacting with the permeability transition pore complex (PTPC). The two anti-apoptotic proteins bcl-2 and bcl-X combine with PTPC, which helps to close PTPC and maintain MMP (Sugrue \& Tatton, 2001). Studies have shown that overexpression of bcl-2 has been observed in CSCs, and the up-regulation of this molecule may partly explain the high MMP phenomenon of TCSCs. (2) Low concentration of ROS. The interaction between ROS and PTPC's sulfhydryl group is beneficial to open PTPC and reduce intracellular MMP (Klein et al., 1996). Some studies have confirmed that embryonic stem cells and hematopoietic stem cells have lower ROS than their progeny cells, and this difference is essential for maintaining stem cell function (Ezashi, 2005; Tothova et al., 2007). The increase of ROS in stem cells can reduce the self-renewal ability of normal stem cells, promote cell differentiation, and reduce cell viability (Naka et al., 2008). In addition, the results of Diehn and others (Diehn et al., 2009) showed that in some tumors, CSCs subgroups have lower ROS levels and stronger ROS resistance than their offspring. This result has also been confirmed in our experiments: LCSCs consume less oxygen and have lower ROS in cells. Therefore, the low concentration of ROS in LCSCs may be another molecular mechanism leading to high MMP. (3) Down-regulation of mitochondrial uncoupling proteins (UCP) (Diehn et al., 2009). UCPs are members of the mitochondrial anion carrier protein family. In mammalian cells, the expression of UCPs is related to negative regulation of MMP. Interestingly, an experiment showed that $\mathrm{UCP}^{-/-}$cells have stronger clonal formation and hypoxia tolerance, more oxygen consumption and low ATP concentration (Derdák et al., 2006). Therefore, the expression level of UCPs may be considered as the third molecular factor of LCSCs high MMP. (4) Low ATP synthase activity. The conversion of ADP into ATP-by-ATP synthase depends on the voltage that consumes MMP (Sugrue \& Tatton, 2001). In other words, the enhancement of ATP synthase activity can be accompanied by a decrease in MMP. Therefore, low ATP concentration may be one of the reasons for the high MMP in LCSCs. (5) Fusion of mitochondria. Studies have found that mitochondrial fusion and MMP also have a certain close relationship. Enlarged mitochondria due to increased fusion and decreased division can maintain high levels of MMP (Zorzano et al., 2010). At the same time, the integrity of MMP also promotes mitochondrial fusion. Reports have proved that MMP downregulation and its exhaustion can completely hinder mitochondrial fusion, and this down-regulation is reversible. When the MMP decreased, the dotted mitochondria can re-fuse and extend to form a connected whole (Ishihara et al., 2003; Song et al., 2007). Although there is no direct evidence to prove these underlying mechanisms in our results, we will further explore the above mechanisms in future research.

Lastly, our results showed that TCSCs consumed less glucose and oxygen, and the concentrations of ATP and ROS were lower than that in non-TCSCs. CSCs consume less $\mathrm{O}_{2}$ and produce less ATP despite increased mitochondrial function. The possible mechanisms may be as follows: (1) cells with "stemness" properties in CSCs are relatively quiescent with low energy demands and (2) CSCs may use substrates other than glucose as energy source. Consistently, a previous study indicated that perinuclear mitochondrial distribution, high MMP, and low amount of mtDNA, ROS and ATP concentrations, and oxygen/glucose consumption were observed in lung CSCs. In addition, large amounts of ROS, which are generated from electron transport within the inner mitochondrial membrane, are produced in the thyroid under physiological conditions (Lee et al., 2015). Diehn and others (Diehn et al., 2009) indicated that subsets of CSCs in tumors enhanced ROS defenses and contained low ROS levels, contributing to radio-resistance. Furthermore, it is well known that cancer cells have higher glucose consumption and lactate production, even under normoxic conditions, than normal cells, which is known as the Warburg effect (Chiaradonna et al., 2006, Ward \& Thompson, 2012).

\section{CONCLUSION}

In summary, alterations in mitochondrial function and cellular energy metabolism can be used as novel indicators of TCSCs.

\section{Consent for publication}

Not applicable.

\section{Competing interests}

The authors declare that they have no competing interests. All authors should confirm their accuracy.

\section{Authors' contributions}

RY and HYL conceived and designed the experiments; XW and ZWC analyzed and interpreted the results of the experiments; YZM performed the experiments; and XLL revised the manuscript.

\section{Acknowledgements}

Not applicable.

\section{REFERENCES}

Bonnet S, Archer SL, Allalunis-Turner J, Haromy A, Beaulieu C, Thompson R, Lee CT, Lopaschuk GD, Puttagunta L, Bonnet S, Harry G, Hashimoto K, Porter CJ, Andrade MA, Thebaud B, Michelakis ED (2007) A mitochondria-K+ channel axis is suppressed in cancer and its normalization promotes apoptosis and inhibits cancer growth. Cancer Cell 11: 37-51. https://doi.org/10.1016/j. ccr.2006.10.020

Brookes PS, Yoon Y, Robotham JL, Anders MW, Sheu SS (2004) Calcium, ATP, and ROS: a mitochondrial love-hate triangle. Am J 
Physiol Cell Physiol. Cell Physiol 287: C817. https://doi.org/10.1152/ ajpcell.00139.2004

Chan DC (2006) Mitochondria: dynamic organelles in disease, aging, and development. Cell 125: 1241-1252. https://doi.org/10.1016/j. cell.2006.06.010

Chiaradonna F, Gaglio D, Vanoni M, Alberghina L (2006) Expression of transforming K-Ras oncogene affects mitochondrial function and morphology in mouse fibroblasts. Biochim Biophys Acta 1757: 13381356. https://doi.org/10.1016/j.bbabio.2006.08.001

Chiaradonna F, Moresco RM, Airoldi C, Gaglio D, Palorini R, Nicotra F, Messa C, Alberghina L (2012) From cancer metabolism to new biomarkers and drug targets. Biotechnol Adv 30: 30-51. https://doi. org/10.1016/j.biotechadv.2011.07.006

Facucho-Oliveira J, John JS (2009) The relationship between pluripotency and mitochondrial DNA proliferation during early embryo development and embryonic stem cell differentiation. Stem Cell Rev Rep 5: 140-158. https://doi.org/10.1007/s12015-009-9058-0

Hernandez BY, Green MD, Cassel KD, Pobutsky AM, Vu V, Wilkens LR (2010) Preview of Hawaii cancer facts and figures 2010. Hawaii Med J 69: 223-224

Kroemer G, Pouyssegur J (2008) Tumor cell metabolism: cancer's Achilles' heel. Cancer Cell 13: 472-482. https://doi.org/10.1016/j. ccr.2008.05.005

Lee J, Chang JY, Kang YE, Yi S, Min HL, Joung KH, Kim KS, Shong M (2015) Mitochondrial energy metabolism and thyroid cancers. Endocrinol Metabol 30: 117-123. https://doi.org/10.3803/ EnM.2015.30.2.117

Lin RY (2011) Thyroid cancer stem cells. Nat Rev Endocrinol 7: 609616. https://doi.org/10.1038/nrendo.2011.127

Liu J, Cao L, Chen J, Song S, Lee IH, Quijano C, Liu H, Keyvanfar $\mathrm{K}$, Chen H, Cao LY, Ahn BH, Kumar NG, Rovira, II, Xu XL, van Lohuizen M, Motoyama N, Deng CX, Finkel T (2009) Bmi1 regulates mitochondrial function and the DNA damage response pathway. Nature 459: 387-392. https://doi.org/10.1038/nature08040

Lonergan T, Brenner C, Bavister B (2006) Differentiation-related changes in mitochondrial properties as indicators of stem cell competence. J Cell Physiol 208: 149-153. https://doi.org/10.1002/ jcp. 20641

Lonergan T, Bavister B, Brenner C (2007) Mitochondria in stem cells. Mitochondrion 7: 289-296. https://doi.org/10.1016/j.mito.2007.05.002

Pan D, Fujimoto M, Lopes A, Wang Y-X (2009) Twist-1 is a PPARdelta-inducible, negative-feedback regulator of PGC-1alpha in brown fat metabolism. Cell 137: 73-86. https://doi.org/10.1016/j. cell.2009.01.051

Piccoli C, Ria R, Scrima R, Cela O, D'Aprile A, Boffoli D, Falzetti F, Tabilio A, Capitanio N (2005) Characterization of mitochondrial and extra-mitochondrial oxygen consuming reactions in human hematopoietic stem cells. J Biol Chem 280: 26467-26476. https://doi. org/10.1074/jbc.M500047200

Straccia P, Santeusanio G, Pierconti F, Brunelli C, Fadda G (2019) Cytologic diagnosis of oncocytic neoplasms of the thyroid gland: The importance of the clinical scenario. Appl Immunobisto M M 27: 726731. https://doi.org/10.1097/PAI.0000000000000713

Toniato A, Boschin I, Casara D, Mazzarotto R, Rubello D, Pelizzo M (2008) Papillary thyroid carcinoma: Factors influencing recurrence and survival. Ann Surg Oncol 15: 1518-1522. https://doi. org/10.1245/s10434-008-9859-4

Visvader JE, Lindeman GJ (2008) Cancer stem cells in solid tumours: accumulating evidence and unresolved questions. Nat Rev Cancer 8: 755-768. https://doi.org/10.1038/nrc2499

Ward PS, Thompson CB (2012) Metabolic reprogramming: a cancer hallmark even warburg did not anticipate. Cancer Cell 21: 297-308. https://doi.org/10.1016/j.ccr.2012.02.014

Witte J, Lehmann S, Wulfert M, Yang Q, Roher HD (2007) Mitochondrial DNA mutations in differentiated thyroid cancer with respect to the age factor. World J Surg 31: 51-59. https://doi.org/10.1007/ s00268-005-0447-5

Xiao-Qun Y, Qi L, Guang-Hui W, Fen-Fen S, Gui-Jun H, Xiu-Wu B, Shi-Cang Y, Gui-Sheng Q (2011) Mitochondrial and energy metabolism-related properties as novel indicators of lung cancer stem cells. Intl J Cancer 129: 820-831. https://doi.org/10.1002/ijc.25944 